\title{
Network Forum
}

\author{
Junli Xu \\ College of Information Engineering, Jiangxi University of Technology, Jiangxi Nanchang
}

Keywords: Network forum; Communicate; Architecture design; Easy maintain

\begin{abstract}
With the wide application and development of computer science and technology, more and more people begin to use computers to communicate and exchange, in which the network forum is an important way to use Internet for information exchange. Network forum system has seven parts including forums classified management, establishment of the forum, subjects publish, forums setting up, personal information services, registration and login and rights management. The multilayer structure set up by the system work makes program structure clear and easy to maintain. The excellent architecture design improves system performance, increases the multiplexing degree of code and at the same time reduces the development difficulty for later programmers.
\end{abstract}

\section{Introduction}

With the popularity of Internet, more and more companies set up their own WWW site, through which the companies can showcase their products, publish the latest developments, exchange and communicate with users, to establish contacts with partners and conduct e-commerce and so on. Network forum system has gradually become an important integral part of the website building, with such a platform that allows users from around the world speak freely on a given topic which facilitates information sharing and learning exchanges with an achievement of brainstorming results. At present, many interactive forums are all explored in the basis of ASP and scripting language, they combine dynamic pages and database together and deal with information through applications, and this is currently the more popular approach.

Designation of this forum is the popularization of combining all kinds of popular network technique and forum and also the forum designed in the basis of ASP.NET technique for enterprises' information management. The forum is to facilitate efficient online communication and dialogue within individuals, groups and between individuals and groups, and at same to help people to carry out activities as learning, communication, online dating 
and so on by taking advantage of internet technology. The establishment of this forum will play a role of bridge and link and greatly facilitate communication between people [1].

The system will generally fulfill three functional parts, which are the member functional part, the moderator functional part and administrator features section.

Firstly, it will introduce the member functional part which is to complete comments on member posts and answers. Members can view all posts and display their comments and opinions on them and can modify their own information.

Secondly, the moderator functional part will be illustrated. In addition to operations for ordinary members, this section also provides the following features, namely the increase, modification, deletion of posts (information within the section only).

Finally, administrator features section is explained. This section provides many functions in which is mainly about management and maintenance of the moderator and posts. The administrator can add, modify and delete information of moderators who can enter relative administration interface only if they have login information allocated by administrators. The administrator can manage all information, and moreover make operation as add, modify, delete and so on.

\section{System Requirements Analysis}

Requirement Analysis. Requirements analysis is a critical step in software design process, which will transform vague concept of software into concrete specification instructions thus laid the foundation for software development.

Functional Requirement.

The main purpose of this system is to provide an information resource exchange platform between the individual and the individual, the team and the team so as to facilitate interpersonal communication and learning. It is according to this characteristic that the system can be divided into three parts. Functional requirement for members is mainly that they can read all articles information and make comments on them. The one for moderators is about issuing subjects to oversight articles posted by members and making addition, modification and deletion for the articles. This part provides many functions in which the main one is to manage and maintain the moderators and the posts (information within the section only). Functional requirement of administrators is mainly that they can add, modify and delete information of moderators who can enter relative administration interface only if they have login information allocated by administrators. The administrator can manage all information, and moreover make operation as add, modify, delete and so on. 
User Requirements.

The system gives users different permissions, the user needs a certain interface. For members, all the information resources of the system will appear on the interface after their login. When members view the information, comments function is available, which means all their operations are for them own when they login. For the moderator, the following permissions are available after their login: post articles view the articles of other members, manage topic posted by their own management, and also log into operation interface of articles posted by members. While for administrators, the corresponding permissions are moderator management, privileged user information management and information resource management. Administrators can change the member into the moderator. They can add, delete and modify the type of information, and then manage information in accordance with the type; they can increase the user account information and make modification and deletion on them.

\section{Database Designation}

Database tables and instructions. This chapter introduces the structure function designation of background database, what will be used at many places in the whole system. People need to call up data from the database, and can complete operations like inserting new data into the database, delete or modify data in the database and so on.

The following are the basic datasheets:

Login Datasheets for Users: majorly record user authentication information

User Ratings Table: major record users' level.

User Registration Datasheets: all information registered by user is mainly recorded.

Datasheets for Forum Type: mainly record the type of forum.

Datasheets for Article Subjects: mainly record the subjects posted by moderators.

Post Information Table: mainly record specific information of posts.

Login Display Table: current user's information display is mainly recorded here.

Details of the database table are showed as follow:

Login Table (Member): record information of login of users mainly to assign different privileges according to the information.

User Ratings List (UserLvRule): record information of users' level to give a certain level record in accordance with the number of users published, as shown in Table 4-2.

User Registration Information Table (UserProfile): record the users' registration information, with the main role of recording all information of users, as shown in Table 4-3. 
Table of Forum Type (Distric): record the type of area, and the main role is to record the type of information the administrator assigned area, as shown in Table 4-4.

Topic Table (Theme): record the theme of the article, the main role is to record the subject information within the section, as shown in Table 4-5.

Post Information Table (Topic): record the information of posts and with the main role of posting specific information recorded under the theme, as shown in Table 4-6.

Login Display List (ForumConfig): mainly record the current user's information and display it on the page, as shown in Table 4-7.

\section{System Designation}

Overall Design for System. The network forum system can be run on Windows 2000 Server operating system platforms, the web servers for it is IIS, and the database server is Microsoft SQL Server2000, with the development tools Microsoft Visual Studio .NET. The workflow of it is: users log in through the authority judging, in which members can browse, comment and publish information while withheld can also operate modification and deletion on the information posted by members except for the operation of ordinary members. The system administrator owns the highest authority as authorizing withheld, setting information block, modifying and editing posts, making improvement on some posts if necessary, deleting some posts that detrimental to the national interest and in violation of the Constitution, and Also an additional treatment for posting user ID if necessary. 


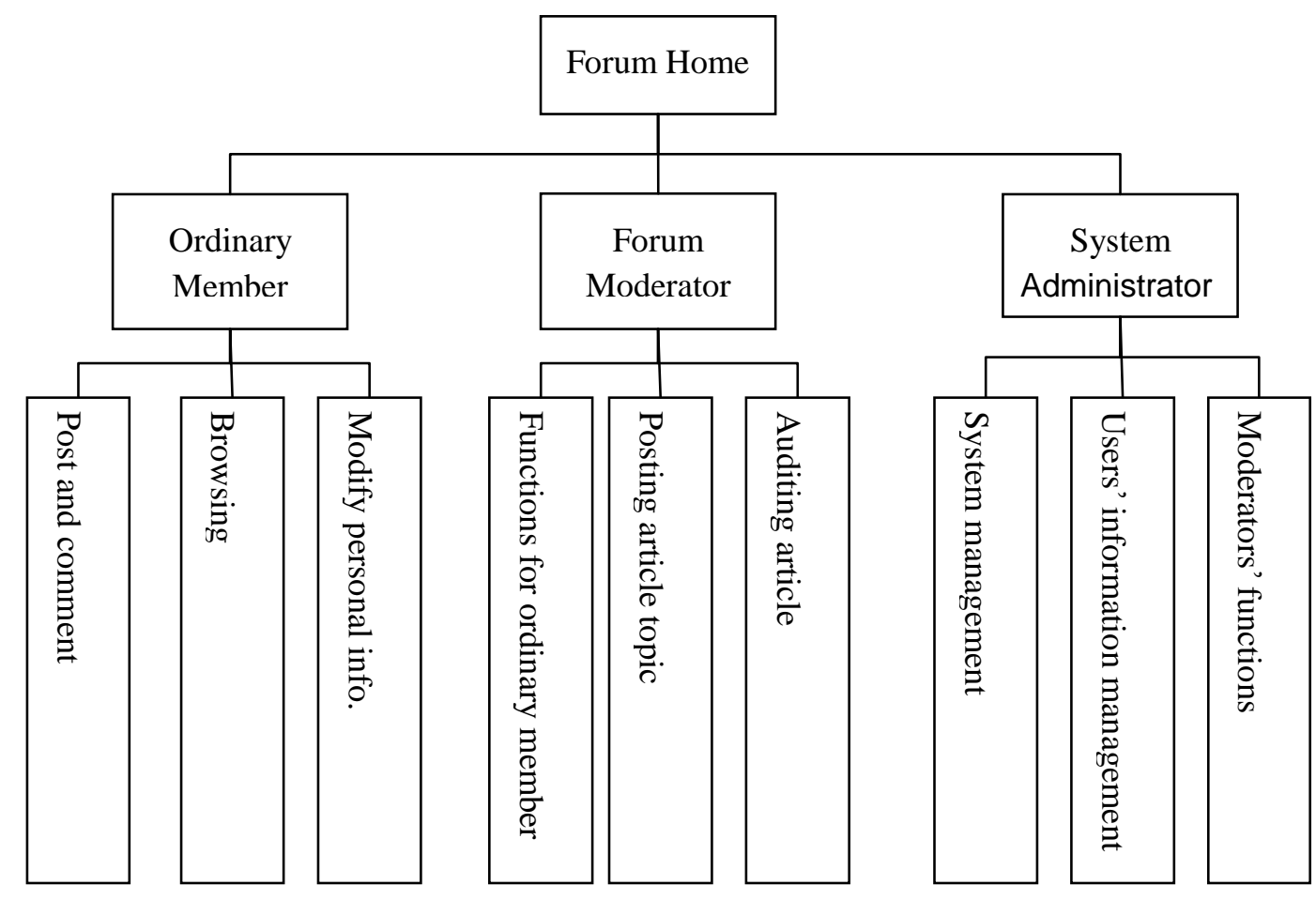

Fig. 1 Functional Structure of the System

Specific design and implement for the system. Main function modules for the system

Specific design process is as follows:

1. System Login: This module is responsible for dividing users into members of user and administrator user to manage user privileges.

2. Skimming: This module is responsible for listing all article information of the Forum in paging, including the title, the type and the release date, title of each piece of information is made to be a hyperlink which can jump pages to read information by clicking on them.

3. Post and Review Articles: This module is responsible for posting and making comments on the articles information.

4. The Theme: The module mainly response to initiate article with theme within the forum for moderators and administrators.

5. Modify and Delete Articles: This module is responsible for moderators and administrator to modify and edit posts, making improvement on some posts if necessary, deleting some posts that detrimental to the national interest and in violation of the Constitution, and Also an additional treatment for posting user ID if needed.

6. User Management: This module is mainly responsible for administrators' operations of authorizing the moderator, modifying and deleting users and so on.

7. System management: This module is mainly responsible for administrators' information management within the forum, forum set and some internal system settings. 
System Function Implement and Major Code.

The basic objective of forum building is to provide the internet users with an interactive, open, easy to use environment by fully use existing network hardware and software resources and WEB open technology.

To achieve this goal, the development and design of application platform must adhere to the subjective requirements of combining content and technology and with content as the priority; not only should it be based on today's advanced technology but also the prospective future technology development requirements should be considered; to maximum adapt to variety environment of hardware and software and take into account different users' characters to make it applicable for general requirements of different users; and to adapt to the market need that get the highest possible efficiency with inputs as low as possible. The current forum system is usually to set up WEB server on the Internet. Users can make operation on website by accessing web site pages.

Viewers can browse the web as a visitor, and if they have interest in the content on the forum, they can register at the forum to become registered users who can post topics and keep abreast.

Users can become a member of the forum through registering in on this interface, then the forum will memorize the user's information and retain articles and topic replies of the users.

Through this interface, administrators can set up and modify the forum area and the subjects in it.

Management of moderators: administrators can authorize and delete user permissions on a certain section of the moderator.

Set the user hierarchy to achieve accumulated experience for users to post. Administrators can adjust the users' level parameters.

At the same time, both of articles' publish and post reply can be submitted to the system via this interface.

Many users divulge passwords for some reasons, but they do not want to let people who know the password use the account, which requires an operation of password modification, shown in Fig. 1.

\section{System Debugging and Testing}

System Debugging. After several months of graduation design, content and function required by the subject has been basically realized. But some places may inevitably be imperfect or omissions. There inevitably exist errors in the procedures, so error handling in 
the development of the project is also very important, and only with a good handling that it will not lead to program interruption or produce some unexpected results because of an error. The more complex the program is, there will be more errors. The whole system is composed by several large modules, the role within which cannot be ignored. People have proposed several techniques to weaken the association between software modules, so that there was less interaction program fragment, but it is just successfully used on some relatively minor problems. In reality of use, there are always a lot of bugs which need to be found through tests and to correct through troubleshooting. Therefore, the author kept debugging and modifying the software after the completion of this subject, so that it can achieve purposes of perfect function and high stability.

\section{Debugging Environment}

WWW server : IIS5.0

Database server : SQL Server 2000

CPU : PIII 850

RAM : $128 \mathrm{M}$

IIS5.0 is a Windows component comes with system of Windows 2000 Server, and shall be installed on the server in the beginning the system configuration. In process of debugging, there has had error as "the system cannot find the path specified", the author solved the problem by reinstalling services manager using Windows 2000 Server installation disk.

Database Debugging. The database is used in almost in all of the operations. In the database, the language used in operation of it is SQL language. SQL language is the standard language of database. In ASP.NET, whenever you want to access a database, the SQL language is a must.

Debugging shall be started with the connection of ASP.NET and database, and the front-end application shall access back-end database by accessing data access interface. ADO is needed in connecting process ASP.NET and database. ADO is an abbreviation for ActiveX Data Object which is one of the most popular methods to access the database at present and a technology that is easy to use and extensible and can add database access into web page. By using ADO, people can write simple scripts to link to Open Database Connectivity (ODBC) -compliant database and OLE DB compliant data source. ADO set inheritance child objects so as to let designers do all kinds of operations to the database, and these inheritance child objects influence to each other and interlocking when ADO is running. There are mainly two objects namely the connection object and records object which in addition have a plurality of sub-objects.

Basic language connecting with database: 
$<\%$ Set conn $=$ Server.CreateObject("ADODB.Connection")

connstr="Provider=SQLOLEDB;Server=(local);Database=database name;UID=login name;pwd=password;"

conn.Open connstr

$\%>$

There have been many problems in the process of add, delete, modify and query, and in modify process, page error always appeared because the author knew little about the way SQL server connect to database at the beginning. After a period of learning about SQL Server, this problem was resolved.

Tests for the System. After the function of the system implementation, tests should be done for the system to verify whether the system can operate normally or not. The test results are as follow:

Section of ordinary members:

When members login the system, they can display simple message for forum on the interface so that users can view and post comments.

Moderators part:

When the moderators $\log$ in, this system can do authentic according to input withheld number or name and password; and after the logging information of the area managed by them will be shown in the interface, and operations like adding, modification, deletion and so on can be implemented successfully.

Administrator components:

When the administrators $\log$ in, this system can do authentic according to input administrator name and password; and after the logging, the administrators can do operations that add and modify information of withheld, area and articles successfully.

\section{Conclusions}

Analysis, design and implement of the Web Forum are basically completed. The functions of it are basically suit to users' needs, and it can carry out functions such as user registration, login, posting articles and administrator management and so on. At the same time, it can provide function of system maintenance which makes users easily do data backup and recovery and data deletion, and moreover makes later maintenance work easy. While the other functions of this system shall be improved to make users' operation and administrators' management and others more convenient and simple. In accordance with users' requirement, this system realizes the entire Forum management by using B/S structure. Users can use the 
system through browser within the network segment permitted with no need to install client software. The user interface can be implemented totally through WWW browser and B/S structure is the preferred one for WEB development. This system exchanges information with the network as its platform. By using this system, information communication between people will be more convenient and faster.

\section{Acknowledgements}

This work was financially supported by project of Technology Department of Jiangxi Province [No 20143BBM26048] and the project of Technology Department of Jiangxi Province [No 2013BBE50051] also gives us lots of help.

\section{References}

[1] Jiang Haipeng, "Effective Control of Network Forum Public Opinion", Qiqihar Daily, 2006 1st phrase (37th in total): page $118-119$.

[2] Stanford H.Rowe, "The 2nd Shenzhen Hi-tech Standardization Forum", China Standardization Foruim, 2006, Vo46 (No.1): page 124 126

[3] Li Wanbao, "Detail Explanation and Application Examples for ASP.NET Technology", and 1st edition, Beijing: Mechanical Industry Press, 2005, page 11-14.

[4]Sun Yongqiang, Dai Feng, Chenzong Bin, "Basis Program Design for Visual C\#.NET", 2nd edition, Beijing: Tsinghua University Press, 2002, page 26-28.

[5] Liu Tao, Lou Xinghua, "SQL Server 2000 Database System Develops Example Navigation”, and first edition, Beijing: People's Posts and Telecommunications Press, 2004, page $45-47$.

[6] Wang Li, "Database Infrastructure and Application”, and second edition, Beijing: China Central Radio and Television University Press, 2002, page 28 to 31.

[7] Qiming Studio, “ASP.NET + SQL Server Network Application Systems Development and Examples", and 1st edition, Beijing: People's Posts and Telecommunications Press, 2005, page 36-39.

[8] Wang Huajie, Huang Shan, "Database Programming", and second edition, Beijing: Science Press, 2003, page 68 to 70. 